\title{
Level Set Topology Optimization of Load Carrying Heat Dissipation Devices
}

\author{
Sandilya Kambampati* \\ University of California San Diego, San Diego, CA 92093, USA \\ Justin S. Gray ${ }^{\dagger}$ \\ NASA Glenn Research Center, Cleveland, $\mathrm{OH} 44135$, USA \\ H. Alicia Kim \\ University of California San Diego, San Diego, CA 92093, USA; Cardiff University, Cardiff, CF24 3AA, UK
}

\begin{abstract}
In this paper, we introduce a level set topology optimization method subjected to coupled mechanical and thermal loads. Examples considering compliance minimization and stress minimization under temperature and volume constraints, and mass minimization under stress and temperature constraints, are presented. The p-norm of the stress field and temperature field is used to approximate the maximum stress and temperature, respectively. The developed method is applied in the design of an L-bracket and a battery package. The results show that designs obtained by ignoring the thermal or structural constraints can result in high values of temperature or stress, respectively.
\end{abstract}

\section{Nomenclature}

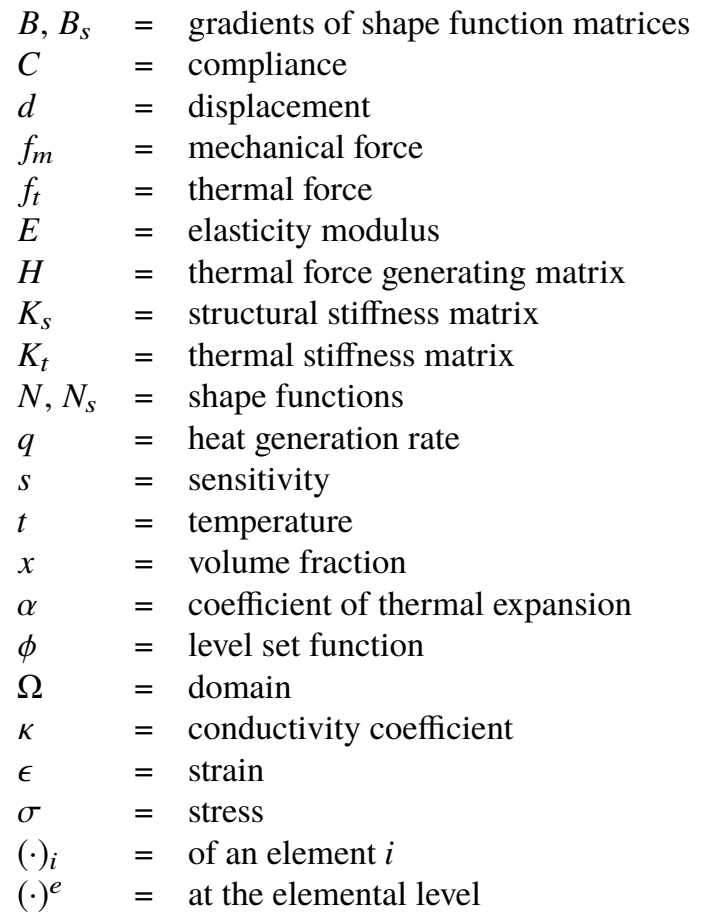

\footnotetext{
*Postdoctoral Research Associate, Department of Structural Engineering, sakambampati@ucsd.edu

$\dagger$ Research Engineer, AIAA Member, justin.s.gray@nasa.gov

¥acobs Scholars Chair Professor, Department of Structural Engineering, Associate Fellow, AIAA, alicia@ucsd.edu
} 


\section{Introduction}

$\mathrm{T}$ HIs paper discusses topology optimization of load carrying heat dissipating devices, typically used in aircraft structures to dissipate heat emanating from batteries, engines, or other heat generating sources [1]. The structures in high temperatures are prone to premature failure due to the combined thermo-mechanical loads. In addition, batteries, especially lithium-ion batteries can experience thermal runway if the working temperature of the battery exceeds a certain limit. The design of such load carrying structures which can also dissipate heat, using level set topology optimization is the focus of this paper.

Topology optimization of thermo-mechanical problems is challenging owing to the design-dependent nature of the thermal loading. Rodrigues and Fernandes [2] presented a material distribution approach to optimize the topology of a 2D solid subject to an increase in temperature. The compliance of the structure is minimized subject to a constraint on the volume. In [3], topology optimization using the evolutionary structural optimization (ESO) algorithm is presented by minimizing the displacement of the structure subjected to mechanical loads and a temperature change. Xia and Wang [4] used the level set method to optimize a topology by minimizing the compliance subject to a volume constraint. They showed that the volume constraint can be inactive for some cases. Gao and Zhang [5] presented a formulation of topology optimization under thermo-elastic stress loads using penalization of the thermal stress coefficient. Zhang et al. [6] optimized for the mean compliance and strain energy minimization under thermoelastic structures, and compared the effects of the a range of objective functions. However, in the above studies, the temperature is assumed to be constant and independent of topology.

In reality, however, the temperature distribution of the structure is dependent on the design in the presence of heat sources or heat sinks due to heat conduction inside the structure. Li et al. [7] presented multi-objective optimization for uniform stress and heat flux distributions of a structure under a given mechanical and thermal loads. Kruijf et al.[8] studied the influence of heat conduction in both structural and material designs. Specifically, Kruijf et al presented a multiobjective topology optimization method, where two conflicting design criteria-the heat conduction and structural stiffness performances are optimized. Deng and Suresh [9] presented stress constrained topology optimization of structures under mechanical and thermal loads. More recently, Kang and James [10] presented multimaterial topology optimization with elastic and thermal response considerations. They conducted parallel uncoupled finite element analyses to simulate the elastic and thermal response of the structure. Zhu et al. [11] presented topology optimization of coupled thermo-mechanical problems by minimizing the compliance of a structure subject to volume and temperature constraints. However, topology optimization of thermo-mechanical structures subject to both temperature and stress constraints is not found in literature.

In this study, we present level set topology optimization of structures under coupled mechanical and thermal loads, subject to stress, temperature, and volume constraints. We conduct a numerical investigation on the designs obtained by minimizing stress and compliance under temperature and volume constraints. The effects of the temperature constraints on the topology of the structure for a range of types of problems are presented. We also discuss the designs obtained by minimizing mass subject to temperature and stress constraints. We discuss the benefits of including both structural and thermal constraints in the optimization process by showing that the designs obtained by ignoring the thermal or structural constraints can result in high values of temperature or stress, respectively.

\section{Optimization Method}

The optimization algorithm presented in this study uses two separate grids: one to represent the level set function and one to conduct the finite element analyses for structural and heat transfer models. The boundary points and the volume fraction information is passed on to the finite element analysis (FEA) mesh. The boundary velocities are optimized using the sensitivity values and mathematical programming, and are passed on to the level set grid. The level set function is updated based on the boundary point velocities by solving a Hamilton-Jacobi equation. In this section, the detailed descriptions of the mathematical models developed are presented.

\section{A. Level Set Method}

In the level set method, the boundary of the structure is described implicitly as [12]

$$
\begin{gathered}
\phi(x) \geq 0, x \in \Omega \\
\phi(x)=0, x \in \Gamma \\
\phi(x)<0, x \notin \Omega
\end{gathered}
$$


where $\phi(x)$ is the implicit level set function, $\Omega$ is the domain, $\Gamma$ is the domain boundary. The boundary of the structure is changed under a given velocity field $V_{n}(x)$ using the following Hamilton-Jacobi equation [13]

$$
\frac{d \phi(x)}{d t}+|\nabla \phi(x)| V_{n}(x)=0
$$

The above equation is solved numerically using the following scheme:

$$
\phi_{i}^{k+1}=\phi_{i}^{k}-\Delta t\left|\nabla \phi_{i}^{k}\right| V_{n, i}
$$

where $i$ is a discrete point in the domain, $k$ is the iteration number, and $\left|\phi_{i}\right|$ is computed using the Hamilton-Jacobi weighted essentially non-oscillatory (HJ-WENO [12]) scheme.

\section{B. Heat transfer model description}

The steady state heat equation [14] is used to model the heat transfer of a structure, given by

$$
-\nabla \cdot(\kappa \nabla T)=q
$$

where $\kappa$ is the conductivity coefficient, $q$ is the heat generation rate, and $T$ is the temperature. The finite element analysis is used to solve the above equation in the discrete form

$$
K_{t} t=q_{t}
$$

where $K_{t}$ is the conductivity matrix, and and $q_{t}$ is the heat generation matrix, given by

$$
K_{t}=\sum_{i=1}^{N_{e}} K_{t i}^{e}=\sum_{i=1}^{N_{e}} \kappa_{i} K_{t 0}^{e}
$$

where $K_{t i}^{e}=\kappa_{i} K_{t 0}^{e}$ is the thermal stiffness matrix of an element $i$, and $K_{t 0}^{e}$ is the homogeneous elemental thermal stiffness matrix given by

$$
K_{t 0}^{e}=\int_{\Omega_{i}} B^{T} B d \Omega
$$

and

$$
q_{t}=\sum_{i=1}^{N_{e}} q_{t i}^{e}=\sum_{i=1}^{N_{e}} \int_{\Omega_{i}} N^{T} q d \Omega
$$

where $N$ is the shape functions, and the gradient $B=\nabla N$ is the gradient of the shape functions, $N_{e}$ is the total number of finite elements, $q_{t}^{e}$ is the elemental heat generation matrix, $\Omega_{i}$ is the domain of an element $i$, and $\kappa_{i}$ is the conductivity coefficient of the element $i$, given by

$$
\kappa_{i}=\kappa_{\min }+x_{i}\left(\kappa_{0}-\kappa_{\min }\right)
$$

where $x_{i}$ is the fraction of the volume of the element cut by the level set, $\kappa_{\min }$ is the conductivity coefficient of the passively conducting material, and $\kappa_{0}$ is the conductivity coefficient of the solid material. The stiffness matrix $K_{t}$ and the heat generation matrix $q_{t}$ are assembled using the above equations and Eq. 5 is solved to determine the temperature distribution.

\section{Thermo-elastic model description}

The temperature distribution causes the structure to expand or contract, resulting in thermal strain $\epsilon_{t}$ due to thermo-elasticity. Specifically, the structural strain $\epsilon_{t, i}$ of an element $i$ caused by the temperature change is given as

$$
\epsilon_{t, i}=\alpha_{i} t_{i}
$$

where $\alpha_{i}=x_{i} \alpha$ is the coefficient of linear expansion of the element $i, \alpha$ is the coefficient of linear expansion of the solid material, and $t_{i}$ is the temperature of the element at the given location. The elemental strain is imposed on the element as a thermal force $f_{t i}^{e}$, given by [2]

$$
f_{t i}^{e}=H_{i}^{e} t_{i}^{e}
$$


where $t_{i}^{e}$ is the temperature at the nodes of an element $i$, and $H_{i}^{e}$ is the elemental thermal load generating matrix given by

$$
H_{i}^{e}=x_{i} H_{0}^{e}
$$

where

$$
H_{0}^{e}=\int_{\Omega_{i}} \alpha B_{s}^{T} C \epsilon^{e} N_{s} d \Omega
$$

and $B_{S}$ is the gradient of the shape function $N_{s}, C$ is the elasticity tensor, and $\epsilon^{e}=[1,0,0,1]^{T}$ in $2 \mathrm{D}$ and $\epsilon^{e}=$ $[1,0,0,0,1,0,0,0,1]^{T}$ is $3 \mathrm{D}$. The elemental thermal load matrix is assembled to form the thermal load matrix as

$$
f_{t}=\sum_{i=1}^{N_{e}} f_{t i}^{e}=H t
$$

where $H$ is the matrix that assembles the thermal force from a given temperature distribution given by

$$
H=\sum_{i}^{N_{e}} H_{i}^{e}
$$

The thermal force is added to the mechanical force $f_{m}$, and the following equation is used to compute the structural displacement $d$ under mechanical and thermal loads

$$
K_{s} d=f_{m}+f_{t}=f_{m}+H t
$$

where $K_{s}$ is the structural stiffness matrix of the structure, given by

$$
K_{s}=\sum_{i=1}^{N_{e}} K_{s i}^{e}=\sum_{i=1}^{N_{e}} E_{i} K_{s 0}^{e}
$$

where $K_{s i}^{e}=E_{i} K_{s 0}^{e}$ is the elemental stiffness matrix of an element $i$, and $K_{s 0}^{e}$ is the homogeneous elemental stiffness matrix, given by

$$
K_{s 0}^{e}=\int_{\Omega_{i}} B_{s}^{T} C B_{s} d \Omega
$$

and $E_{i}$ and $E$ are the elasticity moduli of the element and the material, given by

$$
E_{i}=E_{\min }+\left(E-E_{\text {min }}\right) x_{i}
$$

where $E_{\min }$ is the Elasticity modulus of the void material.

\section{Optimization problem formulation}

The objective of this study is to solve the following optimization problem

$$
\begin{gathered}
\min J=\int_{\Omega} J(\Omega) d \Omega \\
\text { subject to } \\
K_{t} t=q_{t} \\
K_{s} d=f_{m}+H t \\
G_{j}=\int_{\Omega} G_{j}(\Omega) d \Omega \leq g_{j}^{0} \quad j=1,2, \ldots, N_{g},
\end{gathered}
$$

where $J$ is an objective function, $g_{j}$ is the $j^{t h}$ constraint function, $g_{j}^{0}$ is the $j^{t h}$ constraint value, and $N_{g}$ is the number of constraints. The objective function $J$ and the constraint function $G_{j}$ can be compliance, mass, stress, or temperature. The temperature state equations and the thermo-elastic state equations are included as equality constraints in the optimization formulation in Eq. 20 


\section{E. Sensitivity computation}

In this section, the computation boundary point sensitivities for compliance, stress, and temperature, using the adjoint method is presented. First, the sensitivities are computed at the centroids of all the elements from which the boundary point sensitivities are computed using the least squares interpolation [15]. The details of the sensitivity computation is presented here.

\section{Compliance sensitivities}

The compliance of the structure under thermal and mechanical loads is given by

$$
C=d^{T} K_{s} d=f^{T} d=\left(f_{m}+H t\right)^{T} d
$$

The Lagrangian function $\mathcal{L}$ of compliance is defined as

$$
\mathcal{L}=f^{T} d+\lambda_{d}^{T}\left(f_{m}+H t-K_{s} d\right)+\lambda_{t}^{T}\left(f_{t}-K_{t} t\right)
$$

where $\lambda_{d}$ and $\lambda_{t}$ are the adjoint variables corresponding to structural displacement $d$ and temperature $t$. $\lambda_{d}$ is computed by solving $\frac{\partial \mathcal{L}}{\partial d}=0$, which yields

$$
f^{T}-\lambda_{d}^{T} K_{s}=0
$$

Next, $\lambda_{t}$ is computed by solving $\frac{\partial \mathcal{L}}{\partial u}=0$, which yields

$$
\lambda_{d}^{T} H-\lambda_{t} K_{t}=0
$$

The Lagrangian function $\mathcal{L}$ is differentiated with respect to the volume fraction of each element $i$ to compute the elemental centroid sensitivities of compliance $s_{i}$, given by

$$
s_{i}=\frac{\partial \mathcal{L}}{\partial x_{i}}=t_{i}^{e T} H_{0}^{e} d_{i}^{e}+\lambda_{d i}^{e T} H_{0}^{e} t_{i}^{e}-\lambda_{d i}^{e T} K_{s 0}^{e} d_{i}^{e}-\lambda_{t i}^{e T} K_{t 0}^{e} t_{i}^{e}
$$

where $\lambda_{d i}^{e}$ and $\lambda_{t i}^{e}$ are the adjoint variables of displacement and temperature at the element nodes, respectively, $d_{i}^{e}$ and $t_{i}^{e}$ are the displacement and temperature values at the nodes, respectively.

\section{Stress sensitivities}

The maximum stress of a structure is approximated by the p-norm of the stress field. The p-norm of the stress field is given by

$$
\sigma_{p}=\left(\sum_{i=1}^{N e} \sigma_{p, i}\right)^{1 / p}=\left(\sum_{i=1}^{N e} \sum_{j=1}^{N g} \sigma_{v m, i j}^{p}\right)^{1 / p}
$$

where $\sigma_{v m, i j}$ is the Von Mises stress of an element $i$ at a Gauss point $j$, given by

$$
\sigma_{v m, i j}=\sqrt{\sigma_{i j}^{T} V \sigma_{i j}}
$$

where $V$ is the Voigt matrix [16], and $\sigma_{i j}$ is the stress tensor of an element $i$ at a Gauss point $j$, given by

$$
\sigma_{i j}=x_{i} C\left(B_{s, i j} d_{i}^{e}-\epsilon_{t, i j}\right)
$$

where $\epsilon_{t, i j}=B_{t, i j} t_{i}^{e}$ is the thermo-elastic strain, and

$$
B_{t, i j}=\alpha \epsilon^{e} N_{j}
$$

The Lagrangian function $\mathcal{L}$ of the p-norm stress under the structural and thermal equilibrium is given by

$$
\mathcal{L}=\sigma_{p}+\lambda_{d}^{T}\left(f_{m}+H t-K_{s} d\right)+\lambda_{t}^{T}\left(f_{t}-K_{t} t\right)
$$


The adjoint variable $\lambda_{d}$ is computed by solving $\frac{\partial \mathcal{L}}{\partial d}=0$, which yields

$$
f_{\sigma}-\lambda_{d}^{T} K_{s}=0
$$

where $f_{\sigma}=\sum_{i}^{N_{e}} f_{\sigma}^{i}$ and

$$
f_{\sigma}^{i}=\frac{d \sigma_{p, i}}{d d_{i}^{e}}=\frac{\sigma_{p}^{1-p}}{p} \sum_{j=1}^{N g} p \sigma_{v m, i j}^{p-2} \sigma_{i j}^{T} V C B_{s, i j}
$$

The adjoint variable $\lambda_{t}$ is computed by solving $\frac{\partial \mathcal{L}}{\partial t}=0$, which yields

$$
f_{\sigma, t}-\lambda_{t}^{T} K_{t}=0
$$

where $f_{\sigma, t}=\sum_{i}^{N_{e}} f_{\sigma, t}^{i}$ and

$$
f_{\sigma, t}^{i}=\frac{d \sigma_{p, i}}{d t_{i}^{e}}=-\frac{\sigma_{p}^{1-p}}{p} \sum_{j=1}^{N g} p \sigma_{v m, i j}^{p-2} \sigma_{i j}^{T} V C B_{t, i j}
$$

Next the Lagrangian function $\mathcal{L}$ is differentiated with respect to the volume fraction of each element $i$ to compute the pievewise constant sensitivities of the p-norm stress $s_{\sigma, i}$, given by

$$
s_{\sigma, i}=\frac{\partial \mathcal{L}}{\partial x_{i}}=\frac{\sigma_{p}^{1-p}}{p} \sum_{j=1}^{N g}\left[p \sigma_{v m, i j}^{p-2} \sigma_{i j}^{T} C\left(B_{s, i j} d_{i}^{e}-\epsilon_{t, i j}\right)\right]+\lambda_{d i}^{e T} H_{e}^{0} t_{i}^{e}-\lambda_{d i}^{e T} K_{s e}^{0} d_{i}^{e}-\lambda_{t i}^{e T} K_{t 0}^{e} t_{i}^{e}
$$

The sensitivity of the maximum stress is then approximated as

$$
s_{i}=\max _{\forall i, j}\left(\sigma_{v m, i j}\right) \frac{s_{\sigma, i}}{\sigma_{p}}
$$

\section{Temperature sensitivities}

The maximum temperature of the structure is approximated using the p-norm of the temperature vector on the nodes. The p-norm $T_{p}$ of the temperature is given by

$$
T_{p}=\left(\sum_{i=1}^{N_{n}} t_{i}^{p}\right)^{1 / p}
$$

where $N_{n}$ is the number of finite element nodes. The Lagrangian function $\mathcal{L}$ of the temperature under the thermal equilibrium is given by

$$
\mathcal{L}=T_{p}+\lambda_{t}^{T}\left(f_{t}-K_{t} t\right)
$$

The adjoint variable $\lambda_{t}$ is computed by solving $\frac{\partial \mathcal{L}}{\partial t}=0$, which yields

$$
\frac{T_{p}^{1-p}}{p} t^{T}-\lambda_{t}^{T} K_{t}=0
$$

The Lagrangian function $\mathcal{L}$ is differentiated with respect to the volume fraction of each element $i$ to compute the elemental centroid sensitivities of the pnorm of temperature $s_{T, i}$, given by

$$
s_{T, i}=-\lambda_{t i}^{e T} K_{t 0}^{0} t_{i}^{e}
$$

The sensitivity of the maximum temperature is computed as

$$
s_{i}=\max _{\forall i}\left(t_{i}\right) \frac{s_{T, i}}{T_{p}}
$$




\section{Numerical Examples}

In this section, the numerical examples are presented. The compliance and stress of the structures are minimized under a mass constraint and a range of maximum temperature constraints. Next the mass is minimized under stress and temperature constraints, and the resulting designs are compared.

\section{A. L-bracket design}

A schematic of an L-bracket (dimensions are $0.1 \mathrm{~m} \times 0.1 \mathrm{~m}$ and a thickness of $0.01 \mathrm{~m}$ ) is shown in Figure1. A square section of dimensions $0.06 \mathrm{~m} \times 0.06 \mathrm{~m}$ is removed from the top-right side to form the L-bracket. The finite element mesh of size $100 \times 100$ elements is used. A force $F=50 \mathrm{kN}$ is applied on the right hand side and and the L-bracket is clamped on the top. A thermal load $Q=9 \mathrm{~W}$ is applied on the right hand side and the top portion acts as a heat sink. The elastic modulus of the structure is $E=69 \mathrm{GPa}$ and with Poisson's ratio $v=0.3$, density $\rho=2700 \mathrm{~kg} / \mathrm{m}^{3}$, and thermal conductivity $\kappa=235 \mathrm{~W} / \mathrm{m} / \mathrm{K}$.

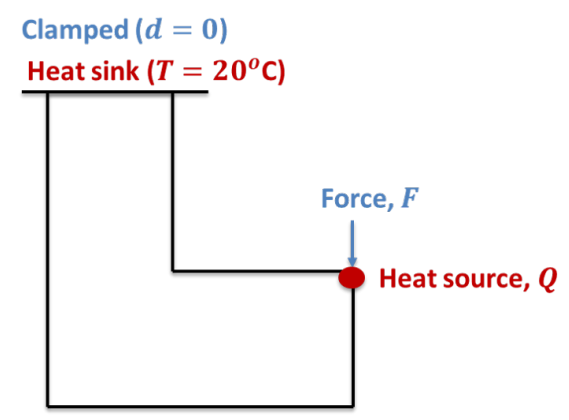

Fig. 1 A schematic of an L-bracket subject to mechanical and thermal loading.

\section{Compliance minimization}

In this section, the compliance $C$ of the L-bracket is minimized subject to a volume constraint $V_{0}=50 \%$ and maximum temperature $T^{*}$ constraints ranging from $49^{\circ} \mathrm{C}$ to $69^{\circ} \mathrm{C}$. The optimization problem can be described as

$$
\begin{gathered}
\min C \\
\text { subject to } \\
K_{t} t=q_{t} \\
K_{s} d=f_{m}+H t \\
V \leq V_{0} \\
T \leq T^{*}
\end{gathered}
$$

Figure 2 shows the optimal topologies obtained for a range of the maximum temperature constraints. Figure 3 shows the compliance values of the optimum topologies obtained for the maximum temperature constraints. As we can see, for low values of maximum temperature constraints, the optimum topology is narrow near the re-entrant corner in order to satisfy the temperature constraint, resulting the high value of compliance. For example when the maximum temperature constraint $T^{*}=49^{\circ} \mathrm{C}$, the optimal compliance is high $(C=906.5 \mathrm{Nm})$. As the maximum temperature constraint is relaxed, the the topology gets wider near the re-entrant corner, and as a result the compliance is reduced. Specifically, for a maximum temperature constraint $T^{*}=69^{\circ} \mathrm{C}$, the optimal compliance is $(C=465.4) \mathrm{Nm}$. In other words, the compliance decreases by a factor of 0.52 when the temperature constraint is increased from $49^{\circ} \mathrm{C}$ to $69^{\circ} \mathrm{C}$. 


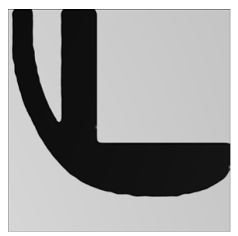

(a) $\mathrm{T}^{*}=49$

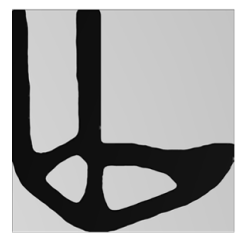

(d) $\mathrm{T}^{*}=61$

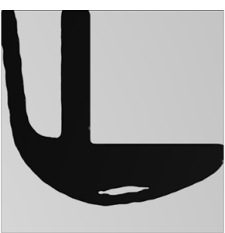

(b) $T^{*}=53$

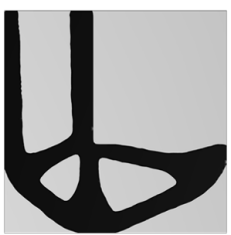

(e) $\mathrm{T}^{*}=65$

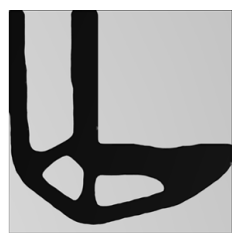

(c) $T^{*}=57$

Fig. 2 Optimal topologies obtained by minimizing structural compliance under a range of maximum temperature constraints.

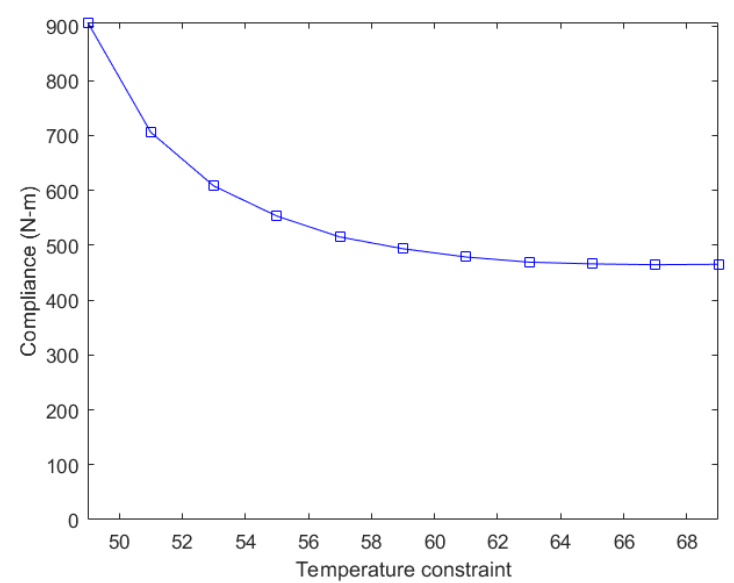

Fig. 3 Optimal compliance of for a range of maximum temperature constraints.

\section{Stress minimization}

In this section, the p-norm stress $\sigma_{p}(p=12)$ of the L-bracket is minimized subject to a volume constraint $V_{0} \leq 50 \%$ and the maximum temperature $T^{*}$ constraints ranging from $49^{\circ} \mathrm{C}$ to $69^{\circ} \mathrm{C}$. The optimization problem can be described as

$$
\begin{gathered}
\min \sigma_{p} \\
\text { subject to } \\
K_{t} t=q_{t} \\
K_{s} d=f_{m}+H t \\
V \leq V_{0} \\
T \leq T^{*}
\end{gathered}
$$

Figure 4 shows the optimal topologies obtained by minimizing the p-norm stress for varying maximum temperature constraints. Figure 5 shows the maximum stress and the p-norm stress values while Figure 6 shows the stress distribution of the optimum topologies obtained for varying maximum temperature constraints.

As we can see from Figure 4, for low values of the maximum temperature constraints, the optimum topology is narrow near the re-entrant corner - in order to satisfy the temperature constraint similar to the compliance minimization 
case, resulting in high stress values. For example in the case of the maximum temperature constraint $T^{*}=49^{\circ} \mathrm{C}$, the optimal topology has a sharp re-entrant corner, and the pnorm stress is high (approximately $140 \mathrm{MPa}$ ).

As the maximum temperature constraint is relaxed, the the topology gets wider and more rounder near the re-entrant corner, and as a result the maximum stress is reduced. Specifically, for the maximum temperature constraint $T^{*}=69^{\circ} \mathrm{C}$, the optimal p-norm stress is $\sigma_{p}=34.7 \mathrm{MPa}$. In other words, the p-norm stress decreases by actor or 0.25 when the temperature constraint is increased from $49^{\circ} \mathrm{C}$ to $69^{\circ} \mathrm{C}$.

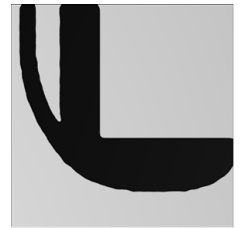

(a) $T^{*}=49$

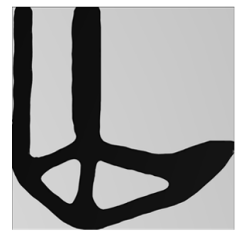

(d) $T^{*}=61$

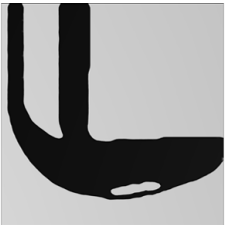

(b) $\mathrm{T}^{*}=53$

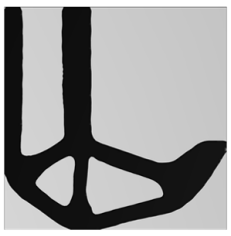

(e) $T^{*}=65$

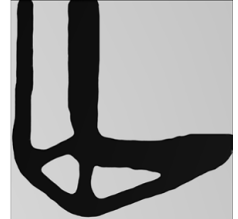

(c) $T^{*}=57$

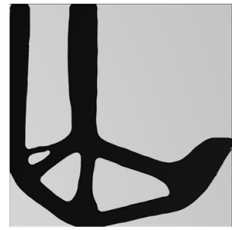

(f) $\mathrm{T}^{*}=69$

Fig. 4 Optimal topologies obtained by minimizing p-norm stress under a range of maximum temperature constraints.

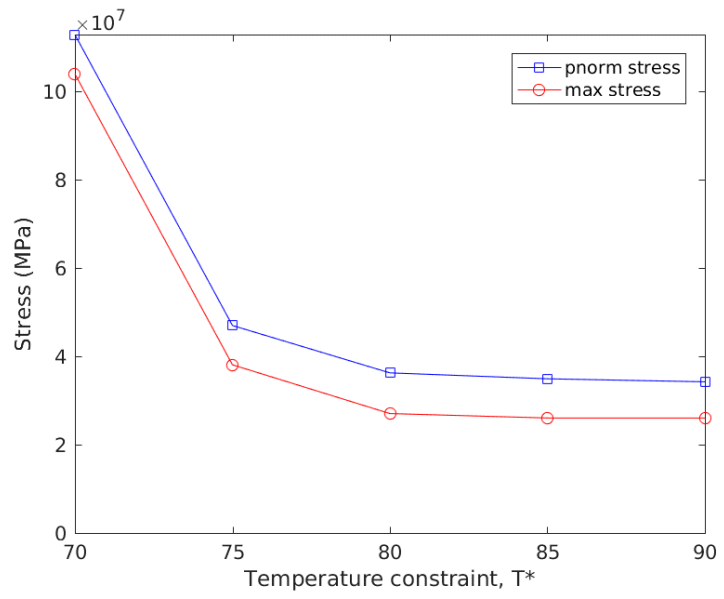

Fig. 5 Optimal p-norm stress and corresponding maximum stress for a range of temperature constraints.

\section{Mass minimization under stress and temperature constraints}

In this section, the mass of the structure is minimized subject to constraints on the maximum stress and temperature. The temperature constraint $T^{*}=65^{\circ} \mathrm{C}$ and the stress constraint $\sigma^{*}=300 \mathrm{MPa}$. The optimization problem can be 


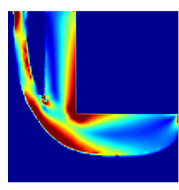

(a) $T^{*}=49$

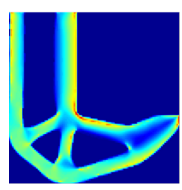

(d) $\mathrm{T}^{*}=61$

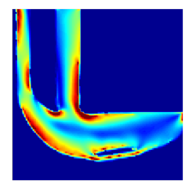

(b) $T^{*}=53$

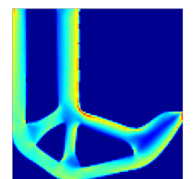

(e) $\mathrm{T}^{*}=65$

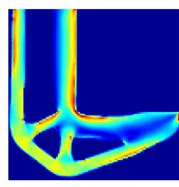

(c) $T^{*}=57$

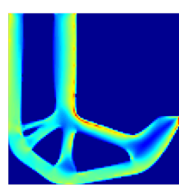

(f) $T^{*}=69$

Stress

(MPa)

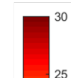

Fig. 6 Stress distribution of optimal topologies obtained by minimizing p-norm stress under a range of maximum temperature constraints.

described as

$$
\begin{gathered}
\min \rho V \\
\text { subject to } \\
K_{t} t=q_{t} \\
K_{s} d=f_{m}+H t \\
\sigma \leq \sigma^{*} \\
T \leq T^{*}
\end{gathered}
$$

The optimal designs are shown in Figure 7 The optimized mass of the structure is $78.3 \mathrm{~g}$, and the temperature and stress constraints are satisfied (top row of Figure 7). When the mass is minimized subject to the stress constraint, the optimal mass $59.4 \mathrm{~g}$ (middle row of Figure 77, which is lower than both stress and temperature constraints. For this case, the stress constraint is satisfied, but the maximum temperature is significantly high $\left(93^{\circ} \mathrm{C}\right)$. On the other hand, when the mass is minimized subject to only the temperature constraint, the optimal mass is $59.9 \mathrm{~g}$ (bottom row of Figure 7), which is also lower than the design case with both stress and temperature constraints. For this case, the temperature constraint is satisfied, but the maximum stress of the structure is high (245 MPa). This investigation shows the importance of considering both the stress and temperature constraints.

\section{B. Battery pack design}

The topology optimization of a battery pack under thermal and mechanical loading is presented. A schematic of a battery pack (dimensions are $10 \mathrm{~cm} \times 10 \mathrm{~cm} \times 5 \mathrm{~cm}$ ) is shown in Figure 8 . The structure is subjected to a uniform loading $\left(\mathrm{F}=10^{7} \mathrm{~N} / \mathrm{m}\right)$ on all four sides. A battery cell is assumed to be non-designable non-load carrying and generates a thermal load of $Q=300 \mathrm{~W} / \mathrm{m}$. The outer part of the structure is assumed to be acting as a heat sink. Due to the symmetry, only a quarter of the structure is modeled using an FEA mesh of $100 \times 100$ elements. The elastic modulus of the structure is $E=69 \mathrm{GPa}$ and Poisson's ratio $v=0.3$, density $\rho=2700 \mathrm{~kg} / \mathrm{m}^{3}$, and thermal conductivity $\kappa=235$ $\mathrm{W} / \mathrm{m} / \mathrm{K}$.

\section{Compliance minimization}

In this section, the compliance of the structure is minimized for a range of maximum temperature constraints from $T^{*}=8^{\circ} \mathrm{C}$ to $T^{*}=15^{\circ} \mathrm{C}$. The optimum topologies are shown in Figure 9 , and the corresponding temperature distributions are shown in Figure 10 .

As we can see, for low values of the maximum temperature constraints, the optimum topology ensures the temperature at the center of the structure is minimized resulting in high values of compliance (Figure 11). For example when the maximum temperature constraint $T^{*}=8^{\circ} \mathrm{C}$, the optimal compliance is high $(C=5.7 \mathrm{kNm})$. As the maximum temperature constraint is relaxed, the the material is more distributed, and as a result the compliance decreases. 


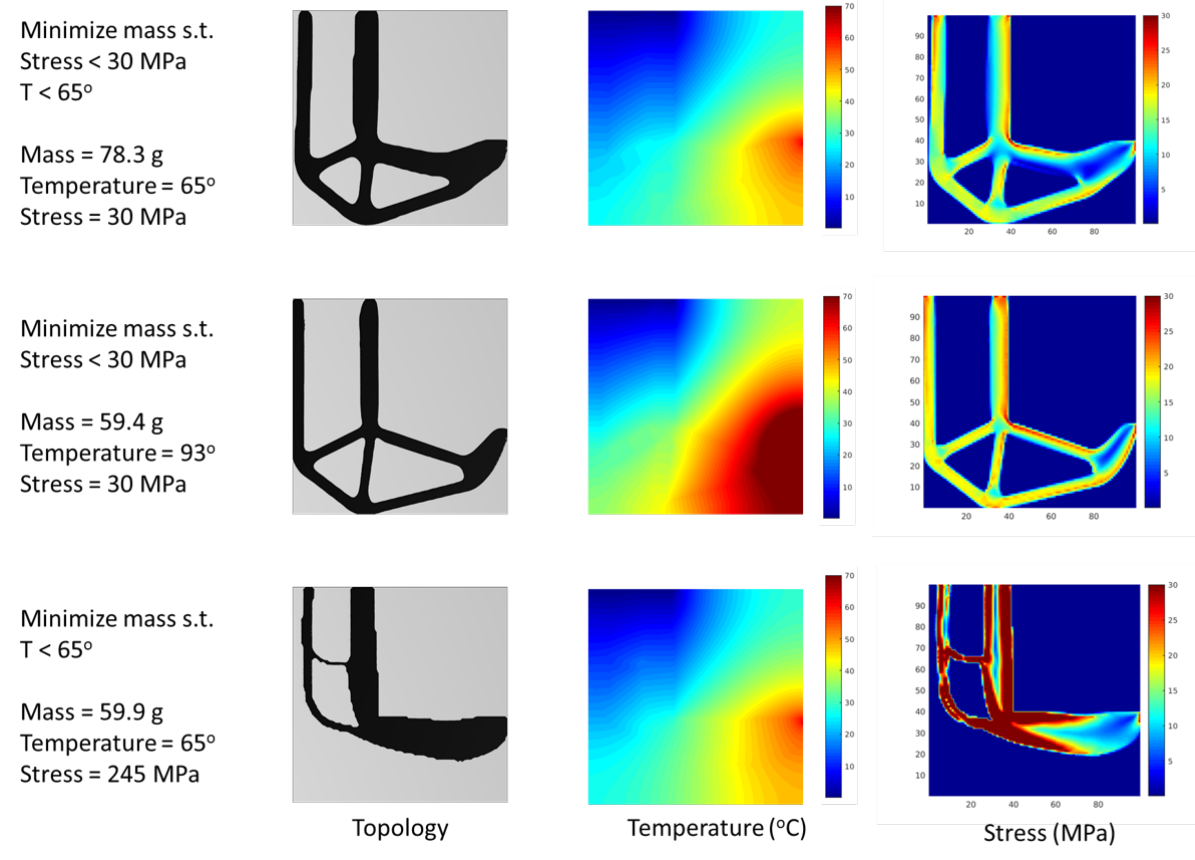

Fig. 7 Minimum mass designs of the L-bracket subject to temperature and stress constraints.

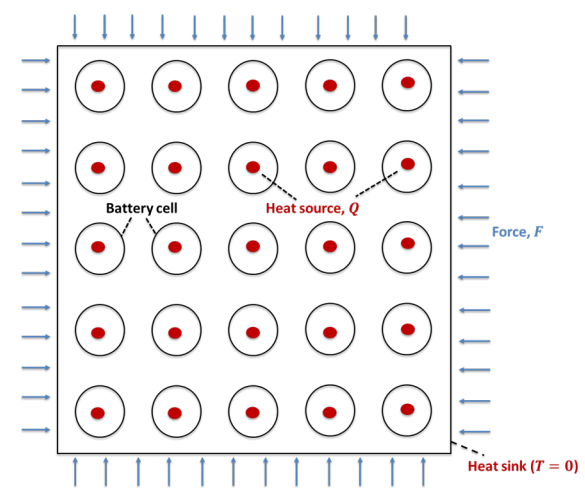

Fig. 8 A schematic of a battery pack subject to mechanical and thermal loading.

Specifically, for the maximum temperature constraint $T^{*}=69^{\circ} \mathrm{C}$, the optimal compliance is $C=1.6 \mathrm{kNm}$. In other words, the compliance decreases by a factor or 0.28 when the temperature constraint is increased from $49^{\circ} \mathrm{C}$ to $69^{\circ} \mathrm{C}$.

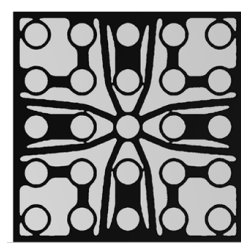

(a) $\mathrm{T}^{*}=8$

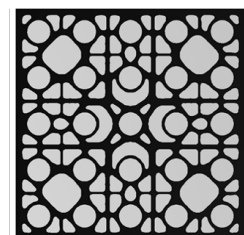

(b) $\mathrm{T}^{*}=11$

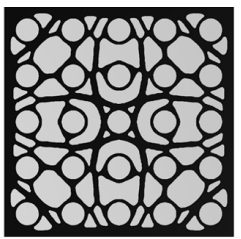

(c) $\mathrm{T}^{*}=14$

Fig. 9 Optimal topologies obtained by minimizing structural compliance under a range of maximum temperature constraints. 


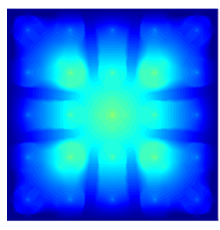

(a) $T^{*}=8$

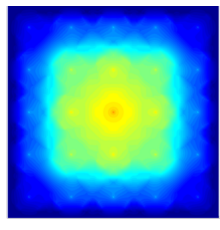

(b) $\mathrm{T}^{*}=11$

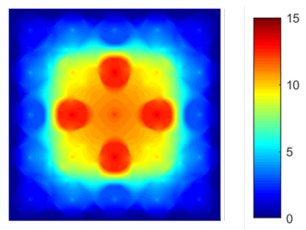

(c) $T^{*}=14$

Fig. 10 Temperature distribution of optimal topologies obtained by minimizing structural compliance under a range of maximum temperature constraints.

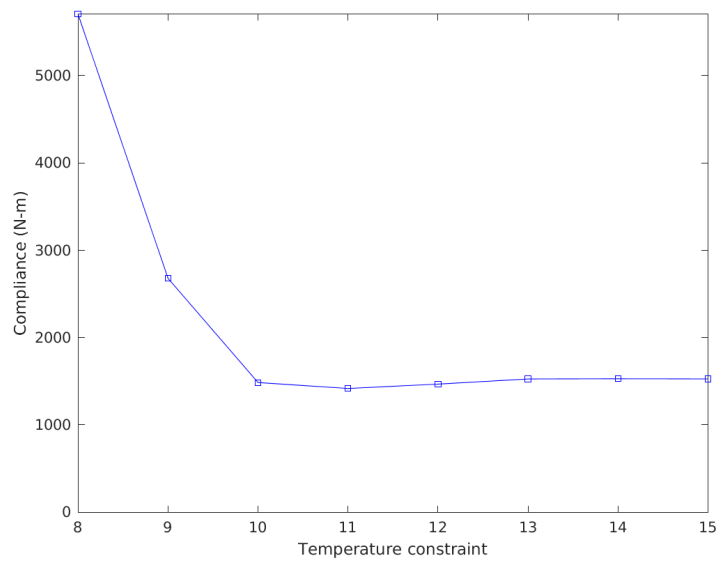

Fig. 11 Optimal compliance for a range of temperature constraints

\section{Stress minimization}

In this section, the p-norm stress of the structure is minimized for a range of maximum temperature constraints from $T^{*}=8^{\circ} \mathrm{C}$ to $T^{*}=15^{\circ} \mathrm{C}$. The optimum topologies obtained are shown in Figure 12 the corresponding temperature distributions are shown in Figure 13, and the stress distribution is shown in Figure 14

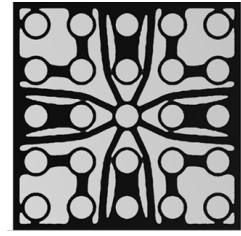

(a) $T^{*}=8$

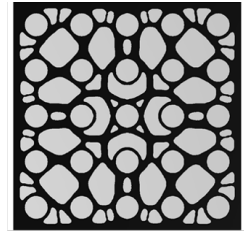

(b) $\mathrm{T}^{*}=11$

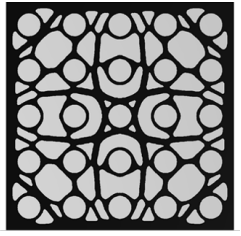

(c) $T^{*}=14$

Fig. 12 Optimal topologies obtained by minimizing structural compliance under a range of maximum temperature constraints.

For the maximum temperature constraints, the optimum topology ensures the temperature at the center of the structure is minimized resulting in high values of stresses (Figure 15). For example when the maximum temperature constraint $T^{*}=8^{\circ} \mathrm{C}$, the optimal p-norm stress is high $\left(\sigma_{p}=470.2 \mathrm{MPa}\right)$. As the maximum temperature constraint is relaxed, the the material is more distributed, and the p-norm stress is lower. Specifically, for the maximum temperature constraint $T^{*}=69^{\circ} \mathrm{C}$, the optimal p-norm stress is $\left(\sigma_{p}=70.0 \mathrm{MPa}\right)$. The p-norm stress decreases by a factor or 0.15 when the temperature constraint is increased from $49^{\circ} \mathrm{C}$ to $69^{\circ} \mathrm{C}$.

\section{Mass minimization subject to stress and temperature constraints}

The mass of the battery pack structure is minimized subject to the constraints on the maximum stress and temperature. The maximum temperature constraint $T^{*}=10^{\circ} \mathrm{C}$ and the stress constraint $\sigma^{*}=100 \mathrm{MPa}$. The optimal designs are 


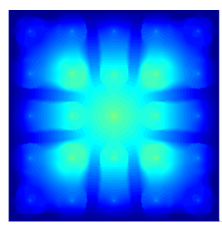

(a) $T^{*}=8$

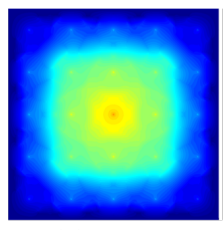

(b) $\mathrm{T}^{*}=11$

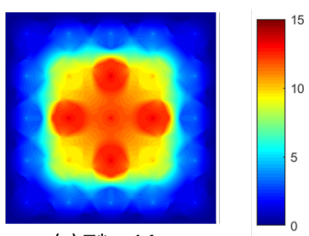

(c) $T^{*}=14$

Fig. 13 Temperature distribution of optimal topologies obtained by minimizing stress under a range of maximum temperature constraints.

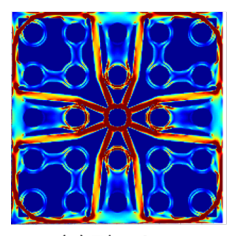

(a) $T^{*}=8$

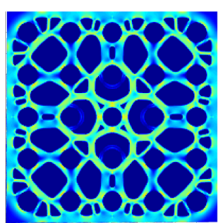

(b) $T^{*}=11$

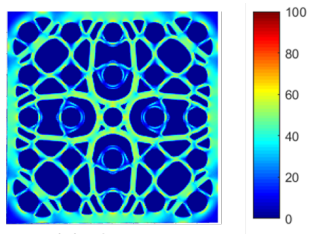

(c) $T^{*}=14$

Fig. 14 Stress distribution (capped at $100 \mathrm{MPa}$ ) of optimal topologies obtained by minimizing p-norm stress under a range of maximum temperature constraints.

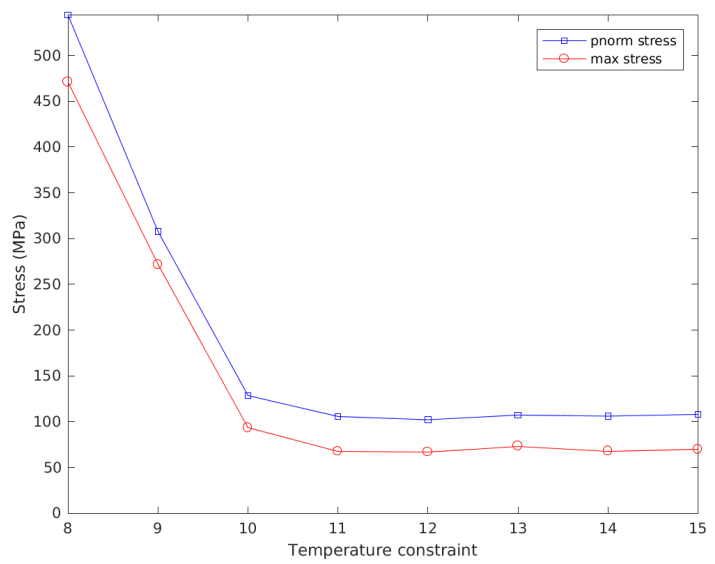

Fig. 15 Optimal p-norm stress and corresponding maximum stress for a range of temperature constraints.

shown in Figure 16. The optimized mass of the structure is $2.27 \mathrm{~kg}$ and the temperature and stress constraints are satisfied (top row of Figure 16. When the mass is minimized subject to only the stress constraint, the optimal mass 1.88 $\mathrm{kg}$ (middle row of Figure 16, which is lower than the design case with both stress and temperature constraints. The stress constraint is satisfied, but the maximum temperature is significantly high, $25^{\circ} \mathrm{C}$. On the other hand, when the mass is minimized subject to only the temperature constraint, the optimal mass is $2.04 \mathrm{~kg}$ (bottom row of Figure 16), which is also lower than the design case with both stress and temperature constraints. The temperature constraint is satisfied, but the maximum stress of the structure is high, $544 \mathrm{MPa}$. This investigation, similar to the L bracket design case, shows the importance of considering both the stress and temperature constraints.

\section{Conclusion}

Topology optimization under coupled mechanical and thermal loads subject to stress and temperature constraints is presented in this paper. The level set method is used for topology optimization, and the level set boundary sensitivities are computed using the adjoint method. The p-norm of the stress and temperature fields is used to approximate the maximum stress and temperature inside the structure, respectively. The designs obtained by minimizing stress and 


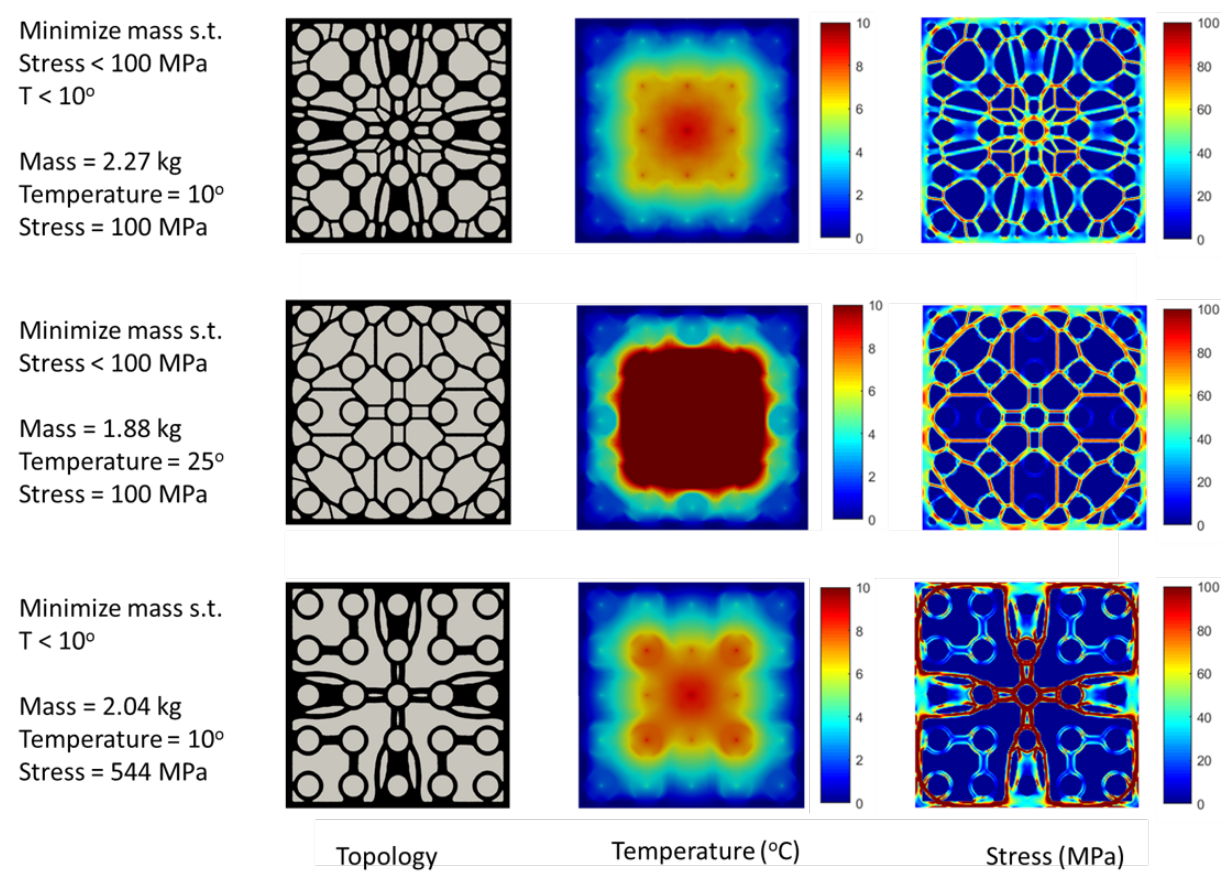

Fig. 16 Maximum stress and temperature constrained minimum mass design.

compliance under temperature constraints show that the compliance and stress can significantly increase for low values of temperature constraints. Additionally, designs obtained by minimizing mass subject to stress and temperature constraints are heavier than the designs obtained by minimizing mass subject to only stress or only temperature constraints. However, the minimum mass designs subject to temperature constraints have high values of stress; and the minimum mass designs subject to stress constraints have high values of temperature. For example, the mass of the L bracket designed for minimum mass subject to only a temperature constraint is $77 \%$ lighter but has a maximum stress value of that is $816 \%$ more than the design obtained by including the stress constraint. Similarly, the mass of the battery pack designed for minimum mass subject to only a temperature constraint is $90 \%$ lighter but has a maximum stress value of that is $544 \%$ more than the design obtained by including the stress constraint. This demonstrates the importance of coupling the thermal and structural analyses in optimization.

\section{Acknowledgments}

The authors acknowledge the support from DARPA (Award number HR0011-16-2-0032) and NASA (grant number 80NSSC18M0153).

\section{References}

[1] Dbouk, T., "A review about the engineering design of optimal heat transfer systems using topology optimization," Applied Thermal Engineering, Vol. 112, 2017, pp. 841-854.

[2] Rodrigues, H., and Fernandes, P., "A material based model for topology optimization of thermoelastic structures," International Journal for Numerical Methods in Engineering, Vol. 38, No. 12, 1995, pp. 1951-1965.

[3] Li, Q., Steven, G. P., and Xie, Y., "Displacement minimization of thermoelastic structures by evolutionary thickness design," Computer Methods in Applied Mechanics and Engineering, Vol. 179, No. 3-4, 1999, pp. 361-378.

[4] Xia, Q., and Wang, M. Y., "Topology optimization of thermoelastic structures using level set method," Computational Mechanics, Vol. 42, No. 6, 2008, p. 837.

[5] Gao, T., and Zhang, W., "Topology optimization involving thermo-elastic stress loads," Structural and multidisciplinary optimization, Vol. 42, No. 5, 2010, pp. 725-738. 
[6] Zhang, W., Yang, J., Xu, Y., and Gao, T., “Topology optimization of thermoelastic structures: mean compliance minimization or elastic strain energy minimization," Structural and Multidisciplinary Optimization, Vol. 49, No. 3, 2014, pp. $417-429$.

[7] Li, Q., Steven, G. P., Querin, O. M., and Xie, Y., "Structural topology design with multiple thermal criteria," Engineering Computations, Vol. 17, No. 6, 2000, pp. 715-734.

[8] de Kruijf, N., Zhou, S., Li, Q., and Mai, Y.-W., “Topological design of structures and composite materials with multiobjectives," International Journal of Solids and Structures, Vol. 44, No. 22-23, 2007, pp. 7092-7109.

[9] Deng, S., and Suresh, K., "Stress constrained thermo-elastic topology optimization with varying temperature fields via augmented topological sensitivity based level-set," Structural and Multidisciplinary Optimization, Vol. 56, No. 6, 2017, pp. $1413-1427$.

[10] Kang, Z., and James, K. A., "Multimaterial topology design for optimal elastic and thermal response with material-specific temperature constraints," International Journal for Numerical Methods in Engineering, Vol. 117, No. 10, 2019 , pp. $1019-1037$.

[11] Zhu, X., Zhao, C., Wang, X., Zhou, Y., Hu, P., and Ma, Z.-D., “Temperature-constrained topology optimization of thermomechanical coupled problems," Engineering Optimization, 2019, pp. 1-23.

[12] Sethian, J. A., Level set methods and fast marching methods: evolving interfaces in computational geometry, fluid mechanics, computer vision, and materials science, Vol. 3, Cambridge university press, 1999.

[13] Sethian, J. A., and Vladimirsky, A., "Fast methods for the Eikonal and related Hamilton-Jacobi equations on unstructured meshes," Proceedings of the National Academy of Sciences, Vol. 97, No. 11, 2000, pp. 5699-5703.

[14] Reddy, J. N., and Gartling, D. K., The finite element method in heat transfer and fluid dynamics, CRC press, 2010.

[15] Dunning, P. D., Kim, H. A., and Mullineux, G., "Investigation and improvement of sensitivity computation using the area-fraction weighted fixed grid FEM and structural optimization," Finite Elements in Analysis and Design, Vol. 47, No. 8, 2011, pp. 933-941.

[16] Zienkiewicz, O. C., Taylor, R. L., and Zhu, J. Z., The finite element method: its basis and fundamentals, Elsevier, 2005. 